\title{
Deep learning models for human centered computing in fog and mobile edge networks
}

\author{
B. B. Gupta ${ }^{1}$. Dharma P. Agrawal ${ }^{2}$. Shingo Yamaguchi ${ }^{3}$
}

Published online: 20 June 2018

c) Springer-Verlag GmbH Germany, part of Springer Nature 2018

Deep learning is a model with multi-level layer structure that uses the underlying output as input from the top. From down to above is a process of the unsupervised learning, which automatically learns useful features, and expresses the lowlevel features as advanced features and from top to bottom is supervised learning process that through the labeled data to the whole network parameter optimization and adjustment of the whole network which has the characteristics of better learning ability. Deep learning has been able to develop so rapidly in recent years mainly due to the following two reasons. (1) The application of massive tagged data mitigates the problem of training. In deep learning, the data is "engine", and Imagenet has millions of annotated data. (2) The rapid development of computer hardware provides a powerful computing power which makes it possible to train large-scale neural networks, such as high-performance GPU can integrate thousands of cores (Jiang et al. 2018; Elmisery et al. 2018; Deng and Yu 2014).

Deep learning models have been proven to be an efficient solution to the most complex engineering challenges. At the same time, human centered computing in fog and mobile edge networks is one of the serious concerns now-a-days. Therefore, it is expected that the development of deep learning based solutions will play an important role for human centered computing in fog and mobile edge networks. This special issue mainly focuses on deep learning models for human centered computing in fog and mobile edge networks and serves as a venue for the researchers around the world to share their state-of-the art research and technological solutions. Topics for this special issue include, but are not

\footnotetext{
B. B. Gupta

bbgupta@nitkkr.ac.in

1 National Institute of Technology Kurukshetra, Kurukshetra, India

2 University of Cincinnati, Cincinnati, USA

3 Yamaguchi University, Yamaguchi, Japan
}

limited to (Li et al. 2018a, b, c; Chong-zhi; Gao et al. 2018; Gupta et al. 2016):

- Deep learning for information revelation and privacy in human centered computing in fog and mobile edge networks

- Deep learning for industrial system in fog and mobile edge networks

- Deep learning for security protocols in human centered computing in fog and mobile edge networks

- Deep learning for fog and mobile edge network modelling and security issues

- Deep learning for security, privacy and management of multimedia data in fog and mobile edge networks

- Deep learning to gain novel insightson in human centered computing in fog and mobile edge networks

- Human centered computing and deep learning concepts and applications

- Deep learning algorithms for learning the behavior analysis in human centered computing in fog and mobile edge networks

- Deep learning for dynamic processes in human centered computing in fog and mobile edge networks

- Deep learning for multimedia data management in fog computing.

This special issue contains eighteen papers focuses on deep learning models for human centered computing in fog and mobile edge networks with and other related areas (Jararweh et al. 2017; Gupta et al. 2018; Stergiou et al. 2018) which were selected after rigorous review process. The first article entitled, "Rational Adversary with Flexible Utility in Secure Two-party Computation" authored by Yilei Wang et al. presents a new kind of rational adversary, who consider payment in his relaxed utilities. The utilities are based on economic incentives instead of standard assumptions. Furthermore, the new rational adversary is assumed to negotiate with rational parties in protocols. It is similar to "cost 
corruption" but more flexible. Proposed new adversary can dynamically negotiate with each rational party in different phases in order to maximize his utilities. To verify the validity of the new adversary, authors model a rational secure two-party protocol, which inherits the hybrid framework of STOC 2007. Authors claimed that they have also proved the security in the presence of the new rational adversary under ideal/real paradigm.

The second paper entitled, "Handling the Adversarial Attacks: A Machine Learning's Perspective", authored by Ning Cao et al. presents a brief introduction of machine learning and adversarial learning, discussing the research frontier of the adversarial issues noticed by both the machine learning and network security field. Authors argue that one key reason of the adversarial issue is that the learning algorithms may not exploit the input feature set enough, so that the attackers can focus on a small set of features to trick the model. To address this issue, authors consider two important classes of classifiers. For random forest, authors propose a type of random forest called Weighted Random Forest (WRF) to encourage the model to give even credits to the input features. This approach can be further improved by careful selection of a subset of trees based on the clustering analysis during the run time. For neural networks, authors propose to introduce extra soft constraints based on the weight variance to the objective function, such that the model would base the classification decision on more evenly distributed feature impact. Empirical experiments show that these approaches can effectively improve the robustness of the learnt model against their baseline systems. The third paper entitled, "A fault-tolerant and energy-efficient continuous data protection system" authored by Quanxin Zhang et al. presents a design method for a fault-tolerant and energy-efficient continuous data protection system (FTEECDP), which is composed of SSD mirror array, TRAP parity generation logic and S-RAID disk array. Taking the SSD mirror array as the source data volume of the system to provide the I/O service of the upper applications, FTEECDP improves the throughput of the system. This method also takes S-RAID as the CDP log volume to store the TRAP parity logs. When the disk data error occurs, the error data will be recovered from the data redundancy information of S-RAID, thus protecting the TRAP parity chain from being destroyed and improving the reliability of the TRAP parity logs. The experiment shows that in the continuous data protection system, the CDP logs stored as the TRAP parity can greatly reduce the storage space overheads and reduce the system energy consumption. In addition, with the disk scheduling algorithm, S-RAID shifts the disk grouping without any data requests into a standby state, thus further reducing the energy consumption of the system.

The fourth paper entitled, "A Novel Character Segmentation Method for Serial Number on Banknotes with Complex
Background" authored by Feng wang et al. presents a novel method which is composed of a hybrid binarization algorithm (HybridB) and an adaptive character extraction algorithm (ACE). The HybridB algorithm utilizes both the global information of the whole image as well as the local information of the pixels which can eliminate the interferences of the noises and background. The ACE algorithm adjusts the boundaries of each character by the local contrast average value information and the distance between its gravity and center, and a decreasing step strategy is also employed to optimize the boundaries to get more accurate segmentation results. To validate the performance of the proposed method, we take experiments on the datasets of RMB, GBP and USD. As authors claim, the binarization and character extraction results show that the proposed method outperforms the other state-of-the-art algorithms in most cases. The fifth paper entitled, "Ontology-based Code Snippets Management in a Cloud Environment" authored by Weixing Ji et al. presents to annotate snippets with a wellformed domain-specific ontology—programming ontology. With a thorough investigation of real world snippets, authors designed a programming ontology to annotate and recommend snippets. In addition, authors built a snippet management system that stores user snippets in the cloud and automatically recommends snippets, so that the retrieval of snippets becomes trivial in popular integrated developing environments, such as Eclipse and Visual Studio. As authors mentioned, the evaluation results demonstrate that the ontology annotation algorithm is able to automatically annotate a snippet with a high degree of accuracy. The shared domain knowledge also makes it possible to share snippets among programmers and systems. As the number of labeled snippets increases, deep learning models can be trained and used to annotate code snippets with high accuracy.

The sixth paper entitled, "Quantitative 3-D Shape Features based Lung Tumor Identification in the Fog Computing Architecture" authored by Jun Xu et al. presents an improved semi-supervised tumor identification method, which takes advantage of the fuzzy c-means clustering algorithm and offers a pathological degree tree based on ten three-dimensional (3-D) and two dimensional (2-D) tumor features. In addition, a great deal of complicated data processing is distributed in the fog computing architecture. First, authors carry out the segmentation of tumors by using FRFCM algorithm, and complete the 3-D modeling. Then, the pathological shape features of 3-D and 2-D tumors are extracted from modeling, for constructing a group of feature vector. Finally, based on the landmark information of labeled samples provided by standard database and experts, authors realize an improved semi-supervised FCM clustering to guide the tumor identification. The experiments are conducted by using medical CT scans of 143 patients including 452 tumors. Overall, the best average identification accuracy 
of $94.6 \%$ has been recorded for this proposed method, the ability of machine learning to recognize the benign, malignant and false-positive tumors is improved effectively under imbalanced data sets. The seventh paper entitled, "A crisis information propagation model based on a competitive relation" authored by Li Yang et al. presents the propagation characteristics of crisis information in different stages and proposes a crisis information propagation model based on a competitive relation. Based on computer simulations of the model, authors identify some influencing factors that affect crisis information propagation and provide some corresponding solution strategies.

The eighth paper entitled, "Framework for replica placement over cooperative Edge Networks" authored by Pingting Hao et al. presents Cloud Co-CDNs (C3), which is a prototype that transfers the management part of edge networks to the cloud with the software-defined network, and cooperation among providers of edge networks is considered in the prototype. Next, authors design a stochastic model for describing the workflow in the $\mathrm{C} 3$ and develop a heuristic algorithm for replica placement to trade off among the latencies that are produced by the delivery process, the updating process and the replication process. Finally, authors evaluate the performances of the proposed algorithms through experimental simulations. Simulation results demonstrate that the proposed algorithms are promising. The ninth paper entitled, "A trust-based collaborative filtering algorithm for e-commerce recommendation system" authored by Liaoliang Jiang et al. presents a slope one algorithm based on the fusion of trusted data and user similarity, which can be deployed in various recommender systems. This algorithm comprises three procedures. First, authors selected trusted data. Second, they calculated the similarity between users. Third, authors added this similarity to the weight factor of the improved slope one algorithm, and then, authors get the final recommendation equation. Authors claim that they have carried out a number of experiments with the Amazon dataset, and the results prove that proposed recommender algorithm performs more accurately than the traditional slope one algorithm.

The tenth paper entitled, "Effective Android Malware Detection with A Hybrid Model Based on Deep Autoencoder and Convolutional Neural Network" authored by Wei Wang et al. presents a hybrid model based on deep autoencoder (DAE) and convolutional neural network (CNN). First, to improve the accuracy of malware detection, authors reconstruct the high-dimensional features of Android applications (apps) and employ multiple CNN to detect Android malware. In the serial convolutional neural network architecture (CNN-S), authors use Relu, a non-linear function, as the activation function to increase sparseness and "dropout" to prevent over-fitting. The convolutional layer and pooling layer are combined with the full-connection layer to enhance feature extraction capability. Under these conditions, $\mathrm{CNN}-\mathrm{S}$ shows powerful ability in feature extraction and malware detection. Second, to reduce the training time, authors use deep autoencoder as a pre-training method of CNN. With the combination, deep autoencoder and CNN model (DAECNN) can learn more flexible patterns in a short time. Authors conduct experiments on 10,000 benign apps and 13,000 malicious apps. CNN-S demonstrates a significant improvement compared with traditional machine learning methods in Android malware detection. In details, compared with SVM, the accuracy with the CNN-S model is improved by $5 \%$, while the training time using DAE-CNN model is reduced by $83 \%$ compared with CNN-S model. The eleventh paper entitled, "HVDB: A Hierarchical Verifiable Database Scheme with Scalable Updates" authored by Zhiwei Zhang et al. firstly presents a new primitive called Vector Commitment Tree (VCT), in which each node is a vector commitment (VC) of its q children. Then, authors utilize VCT as a building block to propose a hierarchical verifiable database scheme (HVDB) with scalable updates, which supports the hierarchical verification and the tampered record localization. Besides, HVDB can also greatly reduce the burden of initialization algorithm of VDB schemes. Finally, the analysis and experimental results show that the proposed HVDB scheme can achieve the desired security requirements and improve the efficiency for practical application.

The twelfth paper entitled, "The Pollutant Concentration Prediction Model of NNP-BPNN Based on the INI Algorithm, AW Method and Neighbor-PCA" authored by Hong Zhao et al. presents the Improved Newton Interpolation (INI) algorithm to solve the problem of missing data, and Assigning Weight (AW) method has been proposed to enrich data of per station. The Neighbor-Principal Component Analysis (Neighbor-PCA) algorithm has been employed to reduce the dimension of data in order to avoid overfitting caused by high dimension and linear correlation of multiple factors. The strategy of early stopping and gradient descent algorithm have been utilized to avoid the slow convergence speed and over fitting by the traditional BPNN. The methods (INI, AW, Neighbor-PCA) have been integrated as a prediction model named NNP-BPNN. Forecasting experiments of PM2:5 have shown that the NNP-BPNN model can improve the accuracy and generalization ability of the traditional BPNN model. Specifically, the average Root Mean Square Error (RMSE) has been reduced by $24 \%$ and the average correlation relevancy has been increased by $9.4 \%$. It took $20 \mathrm{~s}$ to implement BPNN model, it took $170 \mathrm{~s}$ to implement NN-BPNN model and it took $47 \mathrm{~s}$ to implement NNP-BPNN model. The time used by NNP-BPNN model is reduced by $72 \%$ than that of NN-BPNN model. The thirteenth paper entitled, "Allocation Optimization of Pandemic Influenza Antiviral Drugs in Urban Pharmacies" authored by Chijun Zhang et al. presents a network-perspective optimization model across multiple 
social scales (e.g., access, social unbalance, spatial unbalance and resource unbalance) to assign antiviral drugs to the urban dispensing pharmacies. In the network-based frame, authors transfer these considerations to the constraints of group, edge, and node. The constrained optimization model is studied and solved using methods of willingness-to-travel model, L12 norm and network lasso, corresponding to each consideration. Taking Shanghai in a cohort of 11 million individuals as an example, authors have shown the flexibility of the proposed multi-objective model, comparing with the traditional methods. For example, authors found that there are 29 pharmacies needed with covering $81 \%$ districts by tradition single-objective method. In the contrast, only 12 pharmacies are needed with similar access ability but can still cover $75 \%$ districts. Or more pharmacies are assigned with covering $87 \%$ districts.

The fourteenth paper entitled, "Immediate Schedule Adjustment Models and Their Semi-Definite Relaxation in Project Scheduling with Temporary Resource Shortage" authored by Jianping Zhang et al. presents methods for unexpected temporary shortage of some resources during the execution period of a project. Mathematical models for getting immediate adjustment to the existing schedule are established. Semidefinite relaxation technique is also applied to these models for some properly large-scale problems. Relationships between the original models and their semi-definite relaxation problems are analyzed. Finally, some preliminary numerical experiments are performed, and the numerical results show that the proposed models are applicable and feasible to solve the temporary resource shortage problem, and the semi-definite relaxation technique is very helpful to get an immediate schedule adjustment for this kind of problem. The fifteenth paper entitled, "Dynamic management of a Deep Learning-based anomaly detection system for 5G networks" authored by Lorenzo FernándezMaimó et al. presents a MEC-oriented solution in 5G mobile networks to detect network anomalies in real-time and in autonomic way. Proposed approach uses deep learning techniques to analyze network flows and to detect network anomalies. Moreover, it uses policies in order to provide an efficient and dynamic management system of the computing resources used in the anomaly detection process. The paper presents relevant aspects of the deployment of the proposal and experimental results to show its performance.

The sixteenth paper entitled, "Trust Architecture and Reputation Evaluation for Internet of Things" authored by Juan Chen et al. presents IoTrust, a trust architecture that integrates Soft Defined Network (SDN) in IoT, and a crosslayer authorization protocol based on IoTrust. IoTrust and the protocol together provide a new insight for research on trust management in the IoT. For trust establishment, Authors further propose a Behavior-based Reputation Evaluation Scheme for the Node (BES) and an Organization Reputation
Evaluation Scheme (ORES). Both theoretical analysis and simulation results validate the efficiency of BES and ORES. The seventeenth paper entitled, "A Reliable Routing Protocol against Hotspots and Burst for UASN-based Fog systems" authored by Chen Qiuli et al. presents a reliable routing protocol against hotspots and burst (RRAHB). The fuzzy decision algorithm (FDA) for nodes path selecting is designed firstly. When the node chooses the next hop, the candidate nodes are evaluated by FDA. It eliminates the subjectivity of expert scoring and ensure fairness and effectiveness of the evaluated scores. But, FDA cannot solve the problems of hotspots and the load unbalance effectively, which are two important factors that cause data packets loss. Based on FDA, the random selection and hotspots avoidance mechanism are presented. The probability of the hotspots can be reduced by the random selection, and the hotspots effect is eliminated by the hotspots avoidance mechanism. In addition, in order to guarantee the network load balance, a priority-based traffic scheduling mechanism (PTSM) is proposed. It solves the traffic surge problem caused by emergency events and reduces the possibility of packets loss. The simulation results verify the effectiveness of RRAHB in the network with dynamic topology. Compared with the traditional routing algorithm, it has great advantages in improving network reliability and enhancing network performance.

The eighteenth paper entitled, "Sentinel Surveillance of Traffic Conditions with Multilayer Network" authored by Yuan Bai et al. presents a dynamic optimization model of sensor location selection for the sentinel surveillance of dynamical traffic condition under the realistic social and natural environment of a city. Authors use some nonconcave items to model the interactions among sensors, which are the challenge to infer via nonlinear optimization algorithms. As thus, authors give the details of alternating direction method of multipliers algorithm for the proposed model, with the capability to deal with large interactions of variables. Taking the traffic system of Shanghai as a case study, experiments show the proposed model's performance both in accuracy and flexibility of different balance among precision and natural/social constraints.

We would like to express our special thanks to Prof. Vincenzo Loia, the Editor-in-Chief of Journal of Ambient Intelligence and Humanized Computing (AIHC), for his great support and efforts throughout the whole publication process of this special issue. Moreover, this special issue is due to the encouragement of AIHC Editorial office for their continuous support to publish this special issue. Many individuals have contributed toward the success of this issue. Special thanks are due to dedicated reviewers who found time from their busy schedule to review the articles submitted in this special issue. In addition, we are also grateful to all the authors for submitting and improving their papers. 


\section{References}

Deng L, Yu D (2014) Deep learning: methods and applications. Found Trends Signal Process 7(3-4):197-387

Elmisery AM, Sertovic M et al (2018) Cognitive privacy middleware for deep learning mashup in environmental IoT. IEEE Access 6:8029-8041

Gao C-Z, Cheng Q, He P, Susilo W, Li J (2018) Privacy-preserving naive bayes classifiers secure against the substitution-then-comparison attack. Inf Sci. https://doi.org/10.1016/j.ins.2018.02.058

Gupta BB, Agrawal DP, Yamaguchi S (2016) Handbook of research on modern cryptographic solutions for computer and cyber security. IGI Global Publisher, Hershey

Gupta BB, Yamaguchi S, Dharma P, Agrawal (2018) Advances in security and privacy of multimedia big data in mobile and cloud computing. Multimedia Tools Appl 77(7):9203-9208

Jararweh Y et al (2017) Software-defined system support for enabling ubiquitous mobile edge computing. Comput J 60(10):1443-1457

Jiang F, Fu Y, Gupta BB, Lou F, Rho S, Meng F, Tian Z (2018) Deep learning based multi-channel intelligent attack detection for data security. IEEE Trans Sustain Comput. https://doi.org/10.1109/ TSUSC.2018.2793284
Li T, Li J, Liu Z, Li P, Jia C (2018a) Differentially private naive bayes learning over multiple data sources. Inf Sci 444:89-104

Li Y, Wang G, Nie L, Wang Q (2018b) Distance metric optimization driven convolutional neural network for age invariant face recognition. Pattern Recogn 75:51-62

Li J, Sun L, Yan Q, Li Z, Srisa-an W, Ye H (2018c) Significant permission identification for machine learning based android malware detection. IEEE Trans Ind Inf. https://doi.org/10.1109/ TII.2017.2789219

Stergiou C et al (2018) Secure integration of IoT and cloud computing. Future Gen Comput Syst 78:964-975

Zhang Z et al (2016) Social media security and trustworthiness: overview and new direction. Future Gen Comput Syst. https://doi. org/10.1016/j.future.2016.10.007

Publisher's Note Springer Nature remains neutral with regard to jurisdictional claims in published maps and institutional affiliations. 